山्山FFRANÇAISE

$\supset \mathrm{DE}$

$\stackrel{1=1}{\simeq}$ PÉDAGOGIE

\section{Revue française de pédagogie}

Recherches en éducation

182 | 2013

Connaissances et politiques d'éducation : quelles interactions?

\title{
Quels fondements philosophiques pour l'enseignement de la morale laïque ? Pour une éducation au décentrement
}

What philosophical foundations are necessary to teach secular morality? For an education to decentration

Pierre-Étienne Vandamme

\section{(2) OpenEdition}

\section{Journals}

Édition électronique

URL : http://journals.openedition.org/rfp/4026

DOI : $10.4000 /$ rfp.4026

ISSN : 2105-2913

Éditeur

ENS Éditions

Édition imprimée

Date de publication : 28 août 2013

Pagination : 107-116

ISSN : 0556-7807

Référence électronique

Pierre-Étienne Vandamme, «Quels fondements philosophiques pour l'enseignement de la morale

laïque ? Pour une éducation au décentrement », Revue française de pédagogie [En ligne], 182 | 2013, mis en ligne le 28 août 2016, consulté le 20 avril 2019. URL : http://journals.openedition.org/rfp/4026 ; DOI : $10.4000 / \mathrm{rfp} .4026$ 


\title{
Positions, débats et controverses
}

\section{Quels fondements philosophiques pour l'enseignement de la morale laïque? Pour une éducation au décentrement}

\author{
Pierre-Étienne Vandamme
}

Vincent Peillon, ministre de l'Éducation nationale, a suggéré à la rentrée 2012 l'introduction d'un cours de morale laïque à l'école, suscitant les réactions les plus diverses. Cet article examine à la fois la plausibilité d'une éducation morale respectant les exigences de neutralité et de laïcité de l'État et la désirabilité d'un tel enseignement. Distinguant des compréhensions conventionnelle et postconventionnelle de la morale, il conclut à la nécessité sociale d'éduquer à la faculté morale individuelle de décentrement.

Mots-clés (TESE) : éducation morale, laïcité, participation du citoyen, raisonnement, sens critique, politique.

\section{INTRODUCTION}

L'actualité de la rentrée scolaire 2012-2013, en France, a été marquée par la suggestion du ministre de l'Éducation nationale, Vincent Peillon, d'instaurer à l'avenir un cours de morale laïque à l'école, du primaire à la terminale (Fleury, 2012). Cette proposition a suscité les réactions les plus diverses (Le Point, 2012 ; Kosciusko-Morizet, 2012 ; Bauberot, 2012), tantôt encourageantes, tantôt indignées, son prédécesseur décelant même dans les propos du nouveau ministre des relents de " pétainisme ».

Cet article se propose de mettre quelque peu à distance ce débat pour interroger le fond de la question plutôt que sa forme (le nom exact qu'il conviendrait de donner à ce nouveau programme éducatif). Ce faisant, il examinera ce qui nous est apparu comme l'interrogation majeure qu'a suscitée cette proposition : celle qui concerne la tension apparente entre la neutralité requise de la part de l'État et l'enseignement de la morale. Nous commencerons dès lors par un bref rappel des fondements philosophiques de la neutralité de l'État, avant d'examiner quel type de morale peut être enseignée sans enfreindre ce principe de neutralité.

\section{NEUTRALITÉ ET LAIICITÉ DE L'ÉTAT}

Le principe de neutralité et de laïcité de l'État réside au cœur du libéralisme politique. De manière significative, il trouve son origine dans les guerres de religion des $X V I^{e}$ et $X V \|^{e}$ siècles. Dès lors que se différenciaient 
les convictions privées sur la question religieuse, la force de légitimation idéologique du droit divin disparaissait. De ce fait, pour préserver sa légitimité, l'État devait l'asseoir sur d'autres bases (ce sera l'intérêt personnel chez Hobbes, par exemple) et manifester une certaine distance vis-à-vis des diverses convictions privées (Manent, 1987). La notion de tolérance, défendue avec verve par les pères fondateurs du libéralisme politique, John Locke et John-Stuart Mill (Locke, [1689] 2007 ; Mill, [1859] 1987), fut en quelque sorte la réponse politique à la "guerre des dieux ».

Le rôle de l'État se définissant essentiellement par la protection de la liberté individuelle, dans la perspective du libéralisme politique, on en est venu à considérer ce dernier comme "neutre » à l'égard des différentes conceptions de la vie bonne (Rawls, 1991, 1995 ; Dworkin, 1985), ce qui apparut de nouveau, à la fin $d u x^{e}$ siècle, comme la réponse adéquate au pluralisme croissant.

Cependant, cette neutralité prétendue du libéralisme politique a fait l'objet de nombreux débats. La promotion de la liberté individuelle n'est-elle pas elle aussi un parti pris culturel quant à la question de la vie bonne ? Cela est discutable. En effet, s'il est vrai que l'avènement intellectuel du libéralisme trouve son explication dans un contexte particulier, qui fut celui de l'Occident moderne (Rosanvallon, 1989 ; Manent, 1987), la valeur première de la liberté déborde largement ce contexte. II existe en effet deux manières d'interpréter l'émergence du libéralisme politique. L'explication strictement historique la situe dans le contexte des guerres de religion et du développement de l'économie de marché. L'explication pragmatique, elle, y voit la réponse naturelle à la différenciation des visions du monde, au pluralisme naissant. Comment établir un pouvoir légitime dans une société marquée par une diversité de visions du monde également raisonnables? En neutralisant, par l'impartialité, les revendications symétriques à s'imposer comme l'unique modèle de vie bonne, c'est-à-dire en témoignant un égal respect (Dworkin, 1985, p. 191.) à toutes les convictions raisonnables de ce type. Pour être légitime, dès lors, dans une société pluraliste, l'État ne peut qu'être laïc et viser la neutralité quant au bien, objectif qui va inévitablement de pair avec la promotion de la liberté individuelle.

Cela étant, la neutralité et la laïcité de l'État sont également défendables à partir d'une compréhension républicaine de l'État. Cette dernière ne se focalise pas premièrement sur la protection des libertés individuelles face à toute interférence illégitime de l'État ou d'autrui. II ne convient pas, d'un point de vue répu- blicain, de réduire le pouvoir de l'État au strict nécessaire (à déterminer), mais plutôt d'en confier les rênes à tous. Le "gouvernement de tous par tous " (Mill1, [1861] 2009), guidé par une volonté qui « doit partir de tous pour s'appliquer à tous » (Rousseau, [1762] 2008) est la manière dont cette approche prétend fonder la légitimité du pouvoir démocratique.

L'idéal de cette approche est l'unanimité. Le peuple est considéré au singulier, comme une entité homogène, un "corps politique ", habité par une volonté générale. Mais la réalité, c'est la pluralité des valeurs, des convictions et des aspirations individuelles, ainsi que la nécessité d'élire un gouvernement capable d'arbitrer ces revendications concurrentes. Si le pouvoir n'est donc pas effectivement celui de tous, ce qui compte, aux yeux des républicains, est la procédure par laquelle il se conquiert et l'égalité politique (ou "égalité républicaine ») qui la caractérise. Non seulement tous les individus sont égaux devant la loi, mais tous en sont également potentiellement les auteurs et peuvent pour cette raison s'y reconnaître. À cet égard, l'égalité républicaine est une égalité de principe ; elle exige qu'aucun individu ne soit considéré comme ayant plus de valeur qu'un autre. Dès lors, aucune conviction privée ne peut être privilégiée au détriment des autres ; la laïcité s'impose comme condition de possibilité de l'autodétermination républicaine.

Cette laïcité de l'État, cependant, ne peut en aucun cas, sans mettre à mal l'égalité républicaine, privilégier les non-croyants par rapport aux croyants - elle est à comprendre dans le sens d'un " agnosticisme théorique " (Ferry, 2010) plutôt que d'un athéisme de principe. En outre, du fait du pluralisme, le respect de l'égalité républicaine requiert que le « bien commun » poursuivi par l'approche républicaine soit interprété en regard de la distinction libérale entre le juste et le bien, et qu'il se réfère au domaine du juste (plutôt qu'au «bien particulier " éventuellement partagé par la majorité). En effet, si le bien peut être le fruit d'une entente historique sur l'identité qu'une société se donne à elle-même, le juste n'appartient à aucune communauté historique ou culturelle particulière ${ }^{2}$. Pour que tous ses citoyens puissent se reconnaître comme membres d'une même communauté politique, ce que vise le républicanisme, il convient donc que ce dernier accueille en son sein les principes de base du libéralisme politique et que soient satisfaites les exigences de laïcité et de neutralité de l'État. C'est cela même qui le distinguera du communautarisme (Pettit, 2004, p. 160). 


\section{DEUX SENS DU MOT « MORALE »}

Beaucoup des réactions négatives exprimées à propos du projet d'enseigner une morale laïque à l'école découlent d'une interprétation erronée de ce que nous semble devoir désigner, en l'occurrence, le terme "morale ». Dans son sens conventionnel, il se réfère aux mœurs, coutumes et habitudes d'une société donnée. Ainsi, dans les sociétés traditionnelles, enseigner la morale revient à inculquer les normes de comportement ayant la préséance dans la communauté en question. Le contenu de cette morale dépend d'une conception du bien relativement consensuelle. Dans cette perspective, dès lors, l'État devrait abandonner toute prétention à la neutralité s'il se proposait de se faire le garant d'un tel ordre moral.

Dans les sociétés plurales, cependant, l'ordre moral traditionnel s'est vu remis en question par des prétentions dissidentes à la validité morale issues d'autres conceptions raisonnables de la vie bonne. La convention morale s'effritant, une distinction a dû être opérée, afin d'éviter la " guerre des dieux », entre ce qui relève de l'ordre des convictions privées - le bien - et ce sur quoi les citoyens peuvent espérer s'entendre au-delà de leurs divergences de visions du monde - le juste, objet de la raison publique (Rawls, 1995). Une autre manière de différencier les deux termes est d'opposer les questions «comment devons-nous vivre?" - question du bien - et « comment devons-nous vivre ensemble ? »-question du juste (Barry, 2001, p. 77).

Le psychologue L. Kohlberg a qualifié de «postconventionnel » (Kohlberg, 1983) ce stade de développement moral où l'individu est capable de reconnaître l'écart entre droit positif et morale, ainsi qu'entre raisons privée et publique. On peut dès lors qualifier de postconventionnelle une base morale commune, ou en construction, qui est à la fois distincte du droit positif, de la tradition et de toute conception particulière du bien. Au vu de cette distinction, c'est une morale de type postconventionnel qu'il convient d'enseigner, si l'on entend préserver la nécessaire neutralité de l'État.

Étant donné l'importance historique du lien entre morale (conventionnelle) et religion, c'est par extension du terme qu'on parle aujourd'hui de morale «laïque ". Si cette appellation est juste, à certains égards, elle ne doit pas masquer le fait qu'il ne suffit pas à un enseignement de la morale d'être laïc pour être neutre. Mais la question qui se pose alors est la suivante : n'y a-t-il pas incompatibilité fondamentale entre morale et neutralité, même au niveau postconventionnel ?

\section{MORALE ET NEUTRALITÉ}

De la même manière que le contenu d'une morale conventionnelle dépend d'une conception de la vie bonne, celui d'une morale postconventionnelle n'est-il pas indissociable d'une certaine conception du juste? Sans doute. Mais ce constat constitue-t-il une menace envers l'idéal de neutralité ?

Le fondement de la distinction libérale du juste et du bien réside dans l'intuition que les questions de justice, au contraire des questions de sens, sont susceptibles de vérité - on peut qualifier cette approche de " réaliste ". Pour autant, postuler qu'il existe quelque chose de l'ordre d'une vérité morale n'implique nullement de croire que certains possèdent cette vérité (Arneson, 2004, p. 43). II s'agit simplement d'éviter le relativisme moral et de faire droit aux prétentions à la validité qui accompagnent nos jugements moraux quant au juste, tout en préservant la distinction faillibiliste entre consensus et vérité. Sur la base de cette double conception " réaliste " et « faillibiliste " de la morale, la tâche d'un enseignement de la morale n'est pas d'en édicter le contenu, défini une fois pour toutes sur la base d'une décision arbitraire (même si argumentée), mais plutôt de rendre les élèves capables de rationalité morale.

Cependant, la « rationalité morale » est un concept vide si l'on ne possède pas au moins une compréhension générale de ce qu'exige la morale. Ainsi, selon les perspectives philosophiques, on peut identifier la morale avec la poursuite de l'intérêt personnel (Hume, [1739] 1946), avec l'intérêt bien compris (Baier, 1958), l'avantage mutuel (Gauthier, 2000) ou encore l'impartialité (Habermas 1986 ; Barry, 1995).

À partir de là, on peut suivre deux lignes argumentatives différentes. La première part de la défense d'une conception générale du juste prétendant à la supériorité morale sur ses concurrentes (l'impartialité), vise à faire valoir les raisons pour lesquelles on devrait y adhérer et élucide sur cette base les objectifs de l'enseignement de la morale (le décentrement) - dans le cours de morale laïque ou tout autre cours faisant droit à un enseignement de la morale. Cette approche nous paraît philosophiquement adéquate, mais pourrait cependant s'avérer politiquement peu porteuse, semblant reposer sur un nombre trop important de présupposés. La deuxième méthode, que nous proposons ici, consiste à tenter de déceler la conception de la justice sous-jacente à la norme morale postconventionnelle largement dominante aujourd'hui : les Droits de l'homme. 


\section{LA MORALE DES DROITS DE L'HOMME}

Les Droits de l'homme sont le plus souvent critiqués pour les torts qui sont causés en leur nom, pour l'hypocrisie avec laquelle ils sont souvent maniés en politique. Parfois, également, c'est à leur contenu que l'on s'en prend, généralement pour contester leur prétention à l'universalité (en soulignant par exemple leur inscription dans un contexte occidental bien défini), d'autres fois pour critiquer leur non-faisabilité politique. Pourtant, si ces droits n'ont pas véritablement de valeur juridique (par défaut d'une structure politique mondiale, seule capable d'en garantir la protection), ni de fondement philosophique (après tout, ils ne sont que le fruit d'un accord historique négocié entre des élites), ils constituent, d'un point de vue moral, une expérience et une synthèse très instructives.

La force des Droits de l'homme réside en ceci que jamais, à travers les âges, un texte à prétention morale et juridique n'a obtenu autour de lui un tel consensus. Un consensus par recoupement ${ }^{3}$, à vrai dire, car sans que les populations du monde aient eu voix au chapitre dans son élaboration, chaque individu pourrait se retrouver dans le statut qui lui y est accordé. Certes, certains groupes culturels pourront être gênés dans leurs pratiques à cause de la nature de certains droits (la liberté dans le mariage par exemple). Cependant, on conçoit difficilement sur quelles bases autres que des conventions culturelles un individu pourrait raisonnablement (c'est-à-dire en accordant à autrui autant de valeur qu'à soi) en rejeter le contenu. Cela tient à l'individualisme de leur formulation. Mais notre hypothèse est que cet individualisme est inévitable si l'on veut ne point privilégier certains individus au détriment d'autres, si l'on veut faire preuve d'impartialité morale, seul moyen pour une déclaration de se prétendre universelle.

Imaginons ainsi, dans la perspective ouverte par Thomas Scanlon (1982; 1998), que les individus d'un monde entier peu peuplé soient amenés à établir ensemble les principes de base de la société mondiale à construire, et qu'ils soient contraints de sélectionner des principes tels que nul ne puisse raisonnablement les rejeter. Auraient-ils un autre choix que d'établir des droits strictement individuels égaux pour tous ? Comment ces droits pourraient-ils être potentiellement acceptables par tous sans égalité de principe ? L'assemblée délibérative ne serait-elle pas inévitablement raisonnablement contrainte par les plus pauvres de ses membres d'adjoindre aux droits civils et politiques les droits sociaux et économiques, et par les minoritaires à ajouter les droits culturels ?
D'un point de vue moral, cette dimension relativement consensuelle des Droits de l'homme est extrêmement intéressante, car elle laisse présager d'une intuition morale de base largement partagée. En réalité, la plausibilité d'un consensus au sujet des droits humains repose sur la reconnaissance d'une égale dignité de chaque individu, dont l'affirmation est toujours conjointe à celle des droits (Habermas, 2012 , p. 133-135). S'il n'y a sans doute rien de plus vague que l'affirmation générale de l'égale dignité, cette maigre base morale, du moins, semble impossible à rejeter raisonnablement. Pour être plausible (Sen, 1995, p. 16-19), en effet, toute théorie morale postconventionnelle doit pouvoir se justifier sur la base de cette égale dignité et montrer en quoi elle donne une importance égale à chaque individu - définition de l'impartialité morale. On peut dès lors en inférer, sans entrave à la neutralité, que l'impartialité est la base de toute moralité postconventionnelle, ce qui constitue un pas décisif dans l'élucidation des contenus possibles d'un enseignement officiel de la morale.

\section{DÉPASSER LA DIVISION DU TRAVAIL MORAL}

L'idée générale de l'impartialité morale consiste donc à ne pas privilégier quelqu'un, ou certains, au détriment d'autres. Tout comme La Rochefoucauld disait que c'est la crainte de souffrir l'injustice qui fait chérir aux hommes la justice, on peut sans doute dire que c'est la répulsion par tous de l'arbitraire subi qui nous fait reconnaître la légitimité de l'impartialité. Ainsi, nous aspirons probablement tous à mener notre vie comme nous l'entendons, que notre idéal soit orienté par un projet collectif ou individuel. Or, la seule manière de garantir ce droit légitime également pour tous consiste à façonner ce que Rawls appelait la "structure de base de la société " (Rawls, 1991) de telle manière qu'elle ne privilégie personne. À l'égal respect des conceptions du bien et des contextes (linguistiques et culturels) dans lesquels elles se développent s'adjoint donc l'exigence d'égale attention aux opportunités individuelles d'accès aux ressources permettant de mener à bien leur idéal de vie.

Comment s'assurer, cependant, que les normes régissant une société donnée satisfassent cette double exigence d'impartialité ? Par défaut d'un spectateur impartial auquel on pourrait s'en remettre avec confiance, et du fait de la diversité des situations individuelles particulières, ce n'est qu'en donnant la parole à tous qu'on peut s'assurer que personne ne soit 
injustement désavantagé. C'est sans doute là l'intuition fondamentale au cœur des démocraties modernes : afin de ne négliger les intérêts de personne, le droit de participation aux affaires publiques doit être élargi à tous ${ }^{4}$. Le suffrage universel, cependant, n'offre à voir qu'un aperçu des intérêts et aspirations majoritaires. S'il n'était complété par la dimension délibérative de la démocratie - le débat public -, la légitimité de la procédure démocratique serait bien faible. Or, cette dimension délibérative, nécessaire à ce qu'on pourrait appeler la "quête collective d'impartialité ", implique une conception normative forte de la citoyenneté. Être citoyen, ce n'est pas seulement être soumis aux lois ou porteur de droits, c'est également être partie prenante d'une action collective par laquelle une société cherche à sélectionner les normes les plus justes possible.

Ce qui fait que ce n'est plus seulement des institutions qu'on attend l'impartialité, comme le veut la « division du travail moral » (Nagel, 1994, p. 59-68), mais également, dans une certaine mesure, des individus. En effet, dans la conception normative de la démocratie qui semble découler de l'idéal d'impartialité, on attend à la fois des citoyens qu'ils s'expriment pour défendre leurs intérêts personnels si ceux-ci sont injustement bafoués, mais également qu'ils entendent et intègrent les aspirations et objections raisonnables des autres, et enfin se prononcent sur ce que, de leur point de vue - par défaut de " point de vue de nulle part » (Nagel, 1993) -, requiert l'impartialité. Cela ne signifie nullement qu'on attend des citoyens qu'ils se comportent en toutes circonstances de manière impartiale ${ }^{5}$, mais plutôt qu'ils soient capables d'émettre un jugement moral décentré sur les exigences du vivre ensemble, jugement qui doit les pousser à promouvoir et à soutenir des institutions justes.

\section{IMPARTIALITÉ ET DÉCENTREMENT}

Le décentrement (ou la " décentration ") est en effet cette faculté morale par laquelle un individu se montre capable de sortir de soi, d'élargir son jugement pour prendre en compte autrui, en lui accordant autant de valeur qu'à lui-même ${ }^{6}$. Cette démarche intellectuelle est à distinguer de l'altruisme pour deux raisons. Premièrement, elle ne réclame pas l'oubli de soi pour se consacrer uniquement à autrui, mais l'égale attention aux autres qu'à soi en certaines circonstances (mettant en jeu les règles fondamentales du vivre ensemble). Deuxièmement, elle exige un regard critique posé sur soi en plus de l'empathie envers autrui. En effet, on peut aisément imaginer un altruiste qui, convaincu d'une vérité, consacrerait sa vie entière au prosélytisme, fût-il violent, afin de sauver les pauvres âmes de ceux qui ne partagent pas sa foi (Nagel, 1994). Se décentrer implique au contraire d'admettre la possibilité de se tromper, qu'autrui possède la vérité que l'on ne détient pas soi-même, ou qu'aucun des deux ne la possède.

Ainsi apparaît avec évidence la dimension postconventionnelle de la morale du décentrement : elle n'est rien de pré-donné. Sa dimension laïque, elle aussi, découle du même faillibilisme. La reconnaissance de l'agnosticisme théorique, compris dans un sens large, c'est-à-dire l'égale ignorance de tous devant les questions de sens et de foi - attitude de tolérance qui n'empêche pas une « foi critique », basée sur des postulats pratiques plutôt que sur un savoir (Ferry, 2010, p. 102-103) -, apparaît ainsi comme une conséquence du décentrement.

Ce dernier, en effet, est un exercice de raison, bien que son déploiement soit grandement facilité par la présence de sentiments moraux tels que l'empathie, la sympathie, la compassion, la sollicitude ou encore la fraternité. Si l'exigence d'impartialité n'exclut aucunement toutes ces émotions fondamentales, elle requiert un regard critique posé sur nos propensions naturelles à préférer certaines personnes à d'autres. La rationalité, dont il revient aussi à l'enseignant-e de montrer les limites, joue donc un rôle double : 1) de mise à distance des passions et des émotions ; 2) de socle commun permettant à la discussion de s'élever au-dessus des convictions, des vécus et des contextes particuliers, non pas pour les rejeter, mais pour y puiser des arguments susceptibles de recevoir l'assentiment d'autrui.

\section{ÉDUQUER AU DÉCENTREMENT}

II existe de multiples opérations mentales par lesquelles un individu peut se décentrer. La plus intuitive consiste à se mettre à la place d'autrui, exercice facilité par le sentiment plus ou moins spontané d'empathie. Les philosophes en ont inventées d'autres, cependant. D. Hume et A. Smith ont proposé d'imaginer comment un spectateur impartial jugerait nos normes et coutumes (Sen, 2010). Kant ([1785] 1969) a suggéré que la clef de la moralité consiste à universaliser la maxime de son action, afin d'évaluer si l'on pourrait vouloir que tous agissent de la sorte. H. Arendt (1972), inspirée par le Kant de la Critique de la faculté de juger, a vu dans le désintéressement caractéristique de 
l'attitude du spectateur face à une œuvre d'art, et dans la pensée élargie consistant à « courtiser le consentement d'autrui ", les fondements du jugement moral et politique. J. Rawls (1991), dans sa célèbre reprise du contractualisme des Lumières, a développé une situation hypothétique dans laquelle tout individu, ignorant sa position sociale et sa conception de la vie bonne, doit sélectionner les meilleurs principes de justice.

Toutes ces approches, cependant, malgré l'« attitude communicationnelle » (Ferry, 1991, p. 146-147) qu'elles manifestent, demeurent trop monologiques, ne faisant que prêter à autrui des sentiments, des aspirations ou des intérêts. Or, « [a]ucune imputation objective d'utilité ou de coût ne peut remplacer l'appréciation subjective qui en est faite par les intéressés, ainsi que la compréhension intersubjective attendue de la communication mutuelle de cette appréciation. " (Ferry, 1991, p. 174). Pourquoi se passer de la voix d'autrui si on lui porte toute notre attention? Au cœur de cette question repose le projet habermassien d'une éthique de la discussion. Selon cette perspective (Habermas, 1986, 1987, 1992), la communication elle-même, dont la visée est l'entente, possède une fonction « décentrante ", en ce qu'elle pousse à l'adoption de rôles, oblige à comprendre les objections d'autrui, à les intégrer, à réévaluer ses propres convictions, à les délester de ce qui s'est vu raisonnablement rejeter, etc. Au-delà de cette dimension purement " cognitive ", la communication permet aussi un décentrement de l'attention vers les récits d'autrui, la forme narrative étant souvent plus apte que les arguments à exprimer la singularité d'une histoire personnelle et d'une situation, ainsi qu'éventuellement les souffrances qui en découlent (Ferry, 1991, 1996).

Eu égard à ce rôle formateur fondamental de la discussion pour la rationalité morale, l'éducation morale ${ }^{7}$ passe par l'enseignement d'une certaine éthique de la communication. Sa visée serait la "probité » intellectuelle, au sens donné au terme par É. Claparède, c'est-à-dire le respect des principes de non-infaillibilité (chacun peut se tromper) ; nonopportunisme (ne pas adapter ses convictions à ses intérêts) ; équité (réciprocité) ; impartialité (Claparède, cité dans Meirieu, 2005, p. 152) ; auxquels on pourrait ajouter l'égale écoute, l'égale autorité dans la discussion et autres règles de courtoisie discursive.

On est ici dans le registre de l'enseignement de l'argumentation, qui est déjà une des visées fondamentales des cours de français et de philosophie. Cela rend-il superflu un cours de morale spécifique ? Certainement pas. En effet, l'argumentation seule ne suffit pas, d'un point de vue moral. On peut argumenter brillamment des idées atroces, tout comme l'exercice solipsiste de l'argumentation peut renforcer les pires convictions. II faut dès lors, pour que l'argumentation pénètre le champ de la rationalité morale, que lui soient adjoints 1) l'échange, obligeant au décentrement des perspectives, et 2) l'attitude communicationnelle de décentrement, exercée par des expériences de pensée plus «monologiques »: spectateur impartial, pensée élargie, adoption de rôles, réflexion sous voile d'ignorance, etc. Or, face à cela, le professeur de français est démuni, par manque du temps qu'exige l'échange véritable, mais aussi par défaut de formation au décentrement et à l'argumentation morale. Le professeur de philosophie peut se retrouver tout autant démuni, faute de formation spécifique à la rationalité morale. De sorte que souvent, l'exercice scolaire de la dissertation tourne à vide. L'enseignant-e se concentre uniquement sur la forme du raisonnement, ou sur l'appropriation d'idées enseignées, et bien trop peu sur le contenu personnel, prétextant la tolérance de la pluralité des opinions.

Or éduquer au décentrement implique de renoncer au relativisme moral. Ce serait une abdication intellectuelle grave que de se résigner à présenter comme un fait indépassable la pluralité des conceptions du juste et des normes morales. II convient d'abord d'apprendre à distinguer convictions privées et raison publique, puis à confronter les unes aux autres les normes à prétention publique, et les évaluer à l'aune de ce critère normatif décisif qu'est le décentrement : plus une norme est décentrée, plus elle est susceptible de recevoir l'assentiment raisonnable de tous. Pour cette raison, l'enseignant-e ne doit pas se limiter à évaluer la forme d'une argumentation, mais doit chercher à mettre en évidence, pour que l'élève puisse les dépasser, les divers « biais positionnels " (Sen, 2010) et les préjugés qui habitent son propos. En réalité, enseigner l'argumentation n'a rien de moral si la tâche n'est pas accompagnée d'une exigence de décentrement.

\section{ILLUSTRATION CONCRÈTE}

La question pédagogique " comment éduquer au décentrement ? " échappe bien entendu à la portée de cet article, qui examine plutôt les fondements philosophiques d'une telle démarche. Cependant, pour ne pas demeurer uniquement dans l'abstraction et afin d'illustrer les considérations théoriques qui précèdent, nous proposerons, avant de conclure, un exemple de parcours pédagogique concret incarnant ce projet philosophique abstrait. Imaginons ici une classe de 
lycée ${ }^{8}$ au public culturellement et socialement mixte. Profitant de cette circonstance, l'enseignant-e du cours de morale viserait à amener sa classe à produire une réflexion individuelle et collective sur la question des migrations et de la diversité culturelle - enjeu majeur de notre temps.

Le premier exercice proposé serait une production écrite dans laquelle chacun se mettrait à la place d'un autre de la classe, le plus différent possible, en imaginant, pour lui et sa famille, un récit de vie qui expliquerait les raisons pour lesquelles il se trouve aujourd'hui en France et comment il le vit (quel est, par exemple, le regard que la société française pose sur lui et réciproquement). Ensuite, par deux, les élèves ayant échangé leurs positions respectives se regrouperaient et s'échangeraient leurs productions, rétablissant chacun la vérité, la comparant au récit imaginé, en essayant notamment d'identifier ensemble les préjugés culturels ou positionnels (liés à la position socio-économique par exemple) auxquels ils auraient cédé. Dans un second temps, après avoir expliqué aux élèves que le récit seul ne peut faire office d'argument valide dans une discussion ${ }^{9}$, l'enseignant-e leur demanderait de construire des arguments sur la base des récits, en relevant les raisons qu'ils ont identifiées pour expliquer la migration ou la non-migration de l'un et l'autre. Chaque groupe de deux, ayant alors décidé de ce qui serait rapporté à la classe (en excluant, par exemple, ce qui apparaît comme trop intime), présenterait son rapport devant tout le monde. Enfin, la classe tirerait, avec l'enseignant-e, les conclusions générales de l'expérience. On évoquerait, d'une part, les préjugés individuels et culturels, en soulignant à la fois le caractère inévitable des biais positionnels et la possibilité du décentrement. D'autre part, après avoir distingué les apports positifs des migrations et les souffrances liées au phénomène, on examinerait dans leur diversité et leurs tendances lourdes les problèmes qui sont à l'origine et ceux qui sont la conséquence des nombreuses migrations forcées qui caractérisent l'époque.

Le deuxième exercice, un autre jour, consisterait en une argumentation écrite sous voile d'ignorance, où chacun devrait proposer un maximum de trois règles concernant l'organisation du rapport entre les différents pays du monde (sans savoir, donc, auquel il appartient, ni quelle est sa situation sociale) pour remédier aux problèmes qui ont été identifiés dans le premier exercice. Dans la correction de l'exercice, l'enseignant-e veillerait à évaluer le degré de décentrement des arguments et à traquer ce qui, dans les idées émises, repose sur la position (géographique et/ou socio-économique) que l'élève occupe dans la société mondiale. Ces corrections seraient ensuite transmises aux élèves, qui auraient pour tâche de préparer le troisième exercice à venir en reconstruisant ceux de leurs arguments qui auraient été malmenés par l'enseignante ; en les améliorant ou en les modifiant.

Le troisième exercice prendrait la forme d'un débat "public », dans la classe, régulé par l'enseignant- $\mathrm{e}^{10}$, où l'on donnerait successivement la parole à chacun pour proposer les idées formulées seul. Après chaque prise de parole individuelle, on demanderait les réactions constructives de la classe (contre-arguments et non réactions émotives). Tout le monde ayant pris la parole, les élèves, chargés de prendre note des propositions qui leur paraîtraient les plus intéressantes, choisiraient individuellement, mais devant tous, l'idée autre que la leur (ou la leur plus une autre) qu'ils auraient retenue comme la plus intéressante. L'enseignant-e ferait alors apparaître au tableau, pour clôturer le débat, des ensembles regroupant les idées compatibles les plus plébiscitées.

À partir de là, deux issues sont possibles. La première, la plus audacieuse, vise à chercher tous ensemble un consensus raisonnable, selon le modèle scanlonien (Scanlon, 1982, 1998). À charge alors pour l'enseignant-e de définir et d'expliquer la notion de "raisonnable ", selon les critères de réciprocité, d'impartialité et de non-opportunisme. La seconde option, plus aisée, prend acte de l'impossibilité (liée au temps imparti ?) du consensus et fait procéder à un vote, en évoquant les limites de la règle de majorité. Une dernière opération souvent intéressante pour l'enseignant-e consiste alors à demander aux élèves (éventuellement via un questionnaire anonyme, pour encourager la franchise) lesquels ont changé d'avis après le premier ou le second exercice. Cela doit lui permettre soit de tirer des conclusions devant la classe, preuves à l'appui, sur l'importance de la discussion dans la résolution des conflits d'opinion et la quête d'impartialité morale, soit de remettre en question la manière dont le parcours a été mené et d'évaluer si le problème réside dans la procédure ou dans la tension entre l'idéal et le réel, la justice et la démocratie - le fait que nous ne sommes pas égaux et que nous nous accrochons à nos avantages.

\section{UNE ÉDUCATION PUREMENT PROCÉDURALE ?}

À travers cet exemple possible de parcours pédagogique d'éducation au décentrement, il apparaît plus clairement que la neutralité du cours de morale laïque 
découlerait en partie de son caractère procédural. En effet, c'est l'acquisition d'une rationalité morale qui est visée, non l'intégration de contenus moraux substantiels. Bien entendu, l'idéal communicationnel, outil privilégié du décentrement, implique des injonctions substantielles à l'écoute, au respect, à l'honnêteté, etc. Cependant, il n'est jamais fait appel à une conception particulière du bien, ni à une tradition religieuse ou morale déterminée. Toutes les normes sont à disposition des élèves, auxquels il revient d'apprendre à sélectionner les plus adéquates d'un point de vue moral.

Cela ne signifie pas, cependant, que l'éducation à la morale puisse se contenter de cette dimension procédurale. II faut, en effet, inévitablement, la nourrir de contenus (les normes existantes, les débats actuels et passés, les institutions politiques), mais des contenus transmis ${ }^{11}$ de manière informative plutôt que normative. De la sorte, le rappel ou l'enseignement de ces contenus substantiels ne constituera pas une atteinte à la neutralité du cours de morale tant que place sera faite à la pluralité des idées ainsi que des institutions existantes, passées et possibles.

Cette dimension plus " historique " de l'enseignement de la morale, pour être efficace, devra impérativement éviter le piège qui consiste à étudier l'histoire pour elle-même, dans sa simple chronologie, comme si tout ce qu'ont fait et pensé les hommes était intéressant en soi. II faudrait plutôt chercher à mettre en évidence en quoi le passé peut nous permettre de répondre à certaines questions fondamentales qui se posent aujourd'hui, étant un formidable laboratoire d'essais et d'erreurs de l'humanité. Mais contrairement à ce qui se fait le plus souvent dans les cours historiques, la place la plus importante devrait être donnée à l'actualité des débats politiques et moraux - ce qui exige une formation continue des enseignants sur le sujet.

\section{CONCLUSION}

L'objectif de cet article était d'étudier la plausibilité philosophique d'un cours de morale laïque dans l'enseignement public et les fondements qu'il conviendrait de lui donner. À cet égard, la question de la compatibilité entre le projet d'éducation morale et les exigences de neutralité et de laïcité de l'État a été évaluée, et nous avons conclu 1) que la neutralité et la laïcité sont des vertus fondamentales de l'État dans les sociétés pluralistes ; 2) que seul l'enseignement d'une morale " conventionnelle ", ou ancrée dans une conception particulière du bien, serait incompatible avec ces exigences ; 3) qu'un cours de morale laïque devrait viser une approche " postconventionnelle » de la morale, qui opère une distinction entre le juste et le bien ${ }^{12}$, la raison publique et les convictions privées, qui manifeste un égal respect de conceptions raisonnables de la vie bonne, mais maintient le réalisme moral pour les questions de justice. Nous affirmons donc, sur cette dernière base, qu'il est possible d'éduquer à la morale à la fois en visant la neutralité et en refusant le relativisme moral.

Par ailleurs, nous avons jugé qu'en plus d'être possible, l'enseignement officiel de la morale laïque était souhaitable. En effet, si l'impartialité est au fondement de la moralité, comme nous pensons pouvoir l'affirmer, elle est l'idéal que doit poursuivre une société pour être organisée de manière juste. Or, la quête d'impartialité sociale réclame l'investissement de tous dans la procédure démocratique. Et afin que de cette dernière puissent éventuellement émerger des résultats justes, les citoyens doivent acquérir cette capacité morale fondamentale qu'est le décentrement.

Cela signifie que l'éducation morale publique est une nécessité sociale, quel que soit son médium : cours de morale laïque, d'éducation civique ou de philosophie pratique. La pertinence d'introduire un nouveau cours, à cet égard, consiste en ceci qu'on admet le caractère insatisfaisant, sur ce point, des cours déjà existants, et qu'on s'oblige à repenser 1) un programme d'éducation et 2) la formation de ses futurs enseignants.

Pierre-Étienne Vandamme vandammepe@hotmail.com Université catholique de Louvain 
1 Bien que J.-S. Mill soit une figure de proue du libéralisme politique, cette formule qu'il utilise (et emprunte sans doute à Theodore Parker) est au cœur du républicanisme.

2 L'idée que seraient justes des principes tels que nul ne puisse raisonnablement les rejeter, selon la formule de T. Scanlon (1982, 1998) donne une meilleure compréhension de la dimension inachevée de la quête de justice. On peut toujours imaginer que naissent des personnes s'inscrivant dans des situations nouvelles telles qu'elles puissent raisonnablement remettre en question les normes en présence.

3 Par opposition à un consensus « par confrontation », fruit d'une discussion ouverte et argumentée (voir Ferry, 1994).

4 Cela n'est cependant pas suffisant, puisqu'il convient aussi de veiller à égaliser les chances réelles de participation à la procédure démocratique, ce qui implique bien entendu un travail d'éducation et d'égalisation des formations scolaires, mais également une lutte efficace contre l'exclusion sociale.

5 D'abord parce que l'impartialité visée est une « impartialité de second ordre » (Barry, 1995, p. 15), qui admet une sphère de partialité légitime (le fait de passer plus de temps à s'occuper de ses proches que d'inconnus, par exemple). Ensuite parce que l'action collective démocratique ne concerne que la dimension politique de l'existence humaine.

6 Nous avons étudié cette notion plus en détail dans Vandamme, 2012.

7 Je suis redevable à Anne Pelsser d'un travail en commun sur ce thème et d'idées exprimées dans la suite de cet article. Je la remercie de m'avoir autorisé à les présenter ici.

8 Les idées générales proposées sont également valables pour le collège et pour l'enseignement primaire, mais demandent évidemment à être adaptées en fonction de l'âge.
9 S'il peut être très efficace d'un point de vue émotionnel, le récit se révèle inapte à nourrir une argumentation valide. En effet, centré sur une histoire ou une expérience personnelle, on ne peut lui opposer de contre-argument. Dès lors, il est essentiel que les élèves parviennent à placer leurs expériences personnelles au sein d'une réflexion plus globale, qui tendrait à l'universel.

10 Tout ce qu'il-elle peut déléguer aux élèves, à cet égard, est bénéfique. II est en effet préférable d'éviter la posture autoritaire du régent du débat, qui n'existe normalement pas dans le débat public démocratique. Mais il convient évidemment, pour l'exercice, de veiller à la courtoisie discursive, à l'égalité de parole et à la progression du débat.

11 Ceux-ci peuvent avoir été enseignés dans d'autres cours, comme ceux d'histoire ou d'éducation civique, juridique et sociale - avec lesquels doit donc être coordonné le cours de morale.

12 Ce que ne semble pas faire Vincent Peillon, si l'on s'en tient à ses propos rapportés par Le Journal du dimanche : "Cela comporte une construction du citoyen avec certes une connaissance des règles de la société, de droit, du fonctionnement de la démocratie, mais aussi toutes les questions que l'on se pose sur le sens de l'existence humaine, sur le rapport à soi, aux autres, à ce qui fait une vie heureuse ou une vie bonne. " (Fleury, 2012). C'est pour cette raison que certaines personnes, comme Ruwen Ogien, ont dénoncé (à raison, eu égard à la formulation du projet par V. Peillon) un projet " autoritaire " et " inadapté dans sa forme à l'évolution de nos sociétés »(Le Devin, 2012). Notons toutefois que ces propos du ministre précédaient les travaux préparatoires opérés par une commission qu'il a nommée à cet effet, et osons espérer que cette distinction fondamentale lui aura été rappelée.

\section{BIBLIOGRAPHIE}

ARENDT H. (1972). La crise de la culture. Paris : Gallimard.

ARNESON R. (2004). "Democracy is not intrinsically just ». In K. Dowding, R. Goodin \& C. Pateman, Justice and Democracy. Essays for Brian Barry. Cambridge : Cambridge University Press, p. 40-58.

BAIER K. (1958). The Moral Point of View. A Rational Basis of Ethics. New York : Ithaca.

BARRY B. (1995). Justice as Impartiality. A Treatise on Social Justice. Vol. II. Oxford : Oxford University Press.

BARRY B. (2001). Culture and Equality. An Egalitarian Critique of Multiculturalism. Cambridge : Polity Press.

BAUBEROT J. (2012). «Vincent Peillon veut des cours de "morale laïque" : pourquoi c'est une bonne idée ". Le Nouvel Observateur, 3 septembre 2012. En ligne : http:// leplus.nouvelobs.com/contribution/619411-vincentpeillon-veut-des-cours-de-morale-laique-pourquoi-cest-une-bonne-idee.html (consulté le 3 octobre 2012).

DWORKIN R. (1985). A Matter of Principle. Cambridge (États-Unis) : Harvard University Press.

FERRY J.-M. (1991). Les puissances de l'expérience. Essai sur l'identité contemporaine. Tome II. Les ordres de la reconnaissance. Paris : Cerf.

FERRY J.-M. (1994). Philosophie de la communication. Tome II. Justice politique et démocratie procédurale. Paris : Cerf.

FERRY J.-M. (1996). L'éthique reconstructive. Paris : Cerf.

FERRY J.-M. (2010). La religion réflexive. Paris : Cerf.
FLEURY A. (2012). "Peillon : “Je veux qu'on enseigne la morale laïque" ". Le Journal du dimanche, $1^{\text {er }}$ septembre 2012. En ligne : http://www.lejdd.fr/Societe/Education/ Actualite/Vincent-Peillon-veut-enseigner-la-morale-aI-ecole-550018 (consulté le 3 octobre 2012).

GAUTHIER D. (2000). Morale et contrat. Recherche sur les fondements de la morale. Sprimont : Mardaga.

HABERMAS J. (1986). Morale et communication. Conscience morale et activité communicationnelle. Paris : Cerf.

HABERMAS J. (1987). Théorie de l'agir communicationnel. Tome I. Rationalité de l'agir et rationalisation de la société. Paris : Fayard.

HABERMAS J. (1992). De l'éthique de la discussion. Paris : Cerf.

HABERMAS J. (2012). La constitution de l'Europe. Paris : Gallimard.

HUME D. ([1739] 1946). Traité de la nature humaine. Essai pour introduire la méthode expérimentale dans les sujets moraux. T. II. Paris : Aubier.

KANT E. ([1785] 1969). Fondements de la métaphysique des mœurs. Paris : Delagrave.

KOHLBERG L. (1983). Moral Stages. A Current Formulation and a Response to Critics. Bâle (Suisse) : Karger.

KOSCIUSKO-MORIZET N. (2012). « Morale laïque ou instruction civique ". Le Monde, 14 septembre 2012. En ligne : http://www.lemonde.fr/idees/ article/2012/09/14/morale-laique-ou-instruction- 
civique_1760448_3232.html (consulté le 3 octobre 2012).

LE DEVIN W. (2012). “"C'est un projet autoritaire, totalement inadapté." Interview de Ruwen Ogien ". Libération, 4 septembre 2012. En ligne : http://www. liberation.fr/societe/2012/09/04/c-est-un-projetautoritaire-totalement-inadapte_843988 (consulté le 18 mars 2013).

LE POINT (2012). " La morale laïque, pomme de discorde des politiques ". Le Point, 4 septembre 2012. En ligne : http://www.lepoint.fr/societe/lamorale-laique-pomme-de-discorde-des-politiqu es-04-09-2012-1502309_23.php (consulté le 3 octobre 2012).

LOCKE J. ([1689] 2007). Lettre sur la tolérance. Paris : Flammarion.

MANENT P. (1987). Histoire intellectuelle du libéralisme. Dix leçons. Paris : Calmann-Lévy.

MEIRIEU P. (2005). Le choix d'éduquer. Éthique et pédagogie. Paris : ESF.

MILL J.-S. ([1859] 1987). De la liberté. Zürich (Suisse) : Éd. du Grand Midi.

MILL J.-S. ([1861] 2009). Considérations sur le gouvernement représentatif. Paris : Gallimard.

MYERS M. (1983). The Soul of Modern Economic Man. Ideas of Self-interest. Thomas Hobbes to Adam Smith. Chicago : The University of Chicago Press.
NAGEL T. (1993). Le point de vue de nulle part. Paris : Éd. de l'Éclat.

NAGEL T. (1994). Égalité et partialité. Paris : PUF.

PETTIT P. (2004). Républicanisme. Une théorie de la liberté et du gouvernement. Paris : Gallimard.

RAWLS J. (1991). Théorie de la Justice. Paris : Seuil.

RAWLS J. (1995). Libéralisme politique. Paris : PUF.

ROSANVALLON P. (1989). Le libéralisme économique. Histoire de l'idée de marché. Paris : Éd. du Seuil.

ROUSSEAU J.-J. ([1762] 2008). Du contrat social. Paris : Flammarion.

SCANLON T. (1982). "Utilitarianism and Contractualism ». In A. Sen \& B. Williams, Utilitarianism and Beyond. Cambridge : Cambridge University Press, p. 103-128.

SCANLON T. (1998). What We Owe to Each Other. Cambridge (États-Unis) : Harvard University Press.

SEN A. (1995). Inequality Reexamined. Cambridge (ÉtatsUnis) : Harvard University Press.

SEN A. (2010). L'idée de justice. Paris : Flammarion.

VANDAMME P.-É. (2012). Le décentrement. Moralité individuelle et justice sociale. Mémoire de master, philosophie, Université catholique de Louvain (Belgique). 